\title{
Evolutionary Heuristic for Job Scheduling using Grid Computing
}

\author{
Japinder Kaur \\ Dept. of Computer Science \\ GIMET Amritsar
}

\author{
Mehak Naib \\ Dept. of Computer Science \\ GIMET Amritsar
}

\begin{abstract}
Grid is a compilation of huge number of assorted, disseminated, decentralized and energetic resources. Grid computing is a kind of disseminated computing that coordinate and provide the facility of resource sharing on dissimilar environmental location. Resource scheduling in Grid computing is a difficult task due to the heterogeneous and dynamic nature of the resources. This paper includes the comparison of different heuristic approach in grid computing. The presentation of the planned algorithms is evaluated using the GridSim toolkit. The paper reveal the difficulty of the scheduling trouble in Computational Grids when compare to scheduling in standard parallel and distributed systems and show the effectiveness of heuristic and meta-heuristic approaches for the design of efficient Grid scheduler.
\end{abstract}

\section{Keywords}

Resource scheduling, Grid computing, Heuristic approach, Hyper Heuristic approach , GridSim toolkit

\section{INTRODUCTION}

Grid computing is a compilation of computer resources from several locations to achieve a common. Computational Grid is a form of distributed computing that involves coordinating and resource sharing computational authority, data storage and system resources across dynamic and geographically dispersed organization. The aim of Grid computing is to supply the computing facility to users such as power grid without knowing the detailed characteristics of the source. The base of the Grid computing is a resource. To handle varied and energetic nature of resources in the grid environment is monotonous task. Therefore Grid resource management has developed into one of the most important research area in grid computing. The mapping of job to suitable resource for execution of application in Grid computing is NP-Complete problem due to this resource scheduling is significantly challenging task in Grid environment[2][3]. Grid computing combines computers from different organizational domains to solve any task and to achieve a common goal.

In this paper, Section 1 describes the introduction regarding the Grid Computing and Grid Scheduling.

Section 2 contain the related work of the Grid computing and Grid Scheduling.

\section{GRID SCHEDULING}

Computational Grids are the first kind of Grid systems. They were developed due to the need to resolve troubles that need processing a huge amount of operation or data. In spite of the fact that the ability of the computers continue to get better, the computational resources do not react to the continuous demand for additional computational power. Furthermore, lots of statistical studies have shown that computers from companies, organization, etc. are usually underutilized. One of the major objectives of the Computational Grid is, therefore, to advantage from the existence of various computational resources throughout the sharing. The roots of Grid computing can be traced back to the late 1980s and the primary idea that laid the origin of today's Grid systems were developed by researchers from distributed supercomputing for numerical and optimization systems.

Grid scheduling define as the assembly scheduling decision involve allocate job to resources above various managerial domains. A method of identify necessities of the resources matching resources to the application, allocate those resources and lastly scheduling and monitor the Grid resource over time in order to run Grid application as proficiently as possible. To handle resource in Grid environment is a difficult task. hence Grid resource organization has become significant area of Grid computing. Grid resource management system needs to execute resource management decision which include resource scheduling, although maximize the Quality of Service metrics deliver to the customers. Grid scheduling is the most important key challenge of the Grid resource management system since in this, application must be map to the suitable resource although satisfying the user's necessities. The mapping of job to suitable resources for implementation of application in Grid computing is an NP-Complete problem. NP complete problems are often solve via heuristic methods. Heuristic approach can be simply apply to Grid scheduling problem because Grid scheduling has many significant issues like heterogeneity of resources, dynamic and independent nature of Grid resources and lastly the resource supplier and resource user have dissimilar policy for implementation of their application.

The essential grid model usually composed of a lots of hosts, each composed of several computational resources, which may be homogeneous or heterogeneous. In order to accomplish a job require major calculation over a distributed system, it is not easy to have a fair decision on which dispatch technique lead to best result. However, mapping independent task on to a heterogeneous computing (HC) suite is a well known NP Complete Problem[4].

\section{RELATED WORK}

Various Grid scheduling algorithms have been designed that have various features in common, that are perform in different steps to resolve the trouble of matching applications needs with resource accessibility while providing Quality of service(QoS).

Author has developed new heuristic events for the maximum diversity problem (MDP). This NP-hard problem has a major quantity of practical application for example environmental balance, telecommunication services. The algorithm is base on the tabu search method and incorporate memory structure for both structure and development [1]. 
Author proposed genetic algorithm as Search and optimization technique that produce solution to optimization troubles by using technique stimulated by ordinary evolution. This paper presented tentative result of important benchmark function i.e dejong functions by genetic algorithm. This result shows that genetic algorithm give best possible solution [15]. Author proposed that Genetic algorithm is a population-based search and exploit purpose function. Each essential genetic operator use in a simple GA utilizes "random choice" to an extent. Optimization capability can be enhanced when trouble specific knowledge is integrated and objective oriented operators are used[2].

\section{LOCAL SEARCH-BASED HEURISTIC APPROACHES}

\subsection{Genetic Algorithm}

Genetic algorithm is a population-based algorithm. Genetic algorithm works for inclusive optimization. They investigate search space for the discovery of global optimization result by iterative evolution. In genetic algorithm first recognize some terminologies to get insight of the process. There are following steps involved in genetic algorithm. They are: Encoding; initialization; Evaluation, selection, crossover, Mutation and decoding. In encoding the problems is solved by using genetic algorithms. In this state, phenotype is mapped to genotype. The second step that is initialization take input parameter such as population size, mutation probability etc. Then the next step i.e. evaluation, finds the fitness values of individuals by using fitness function. Selection step retain best fit individual and eliminate all the bad population. Mutation is used to add the new features in the gene. The last and final step of genetic algorithm is decoding means decode the final and absolute solution back to the phenotype[4].

\subsection{Hill Climbing}

Hill Climbing (HC) is a graph search algorithm where the existing path is extensive with a successor node which is nearer to the result than the conclusion of the existing path. Hill climbing is rational and advantageous mainly in situation where the exploration space is of straightforward environment with no more than a particular maxima or minima. $\mathrm{HC}$ is a local search heuristic technique. Hill climbing is simpler and straight forward in comparison to other heuristics.

\subsection{Simulated Annealing}

Simulated Annealing (SA) heuristic approach was proposed by kirkpatrick et al in 1983 . The simulated annealing process consists of first melting the system being optimized at high efficient temperature, then lower the temperature by slow stages until the system freezes and no further changes occur. SA is guaranteed to converge in asymptotic time. SA statically guarantees to find an optimal solution.

SA is a robust heuristic to implement and has an ability to provide reasonably good solutions formany combinatorial problems. SA is comparatively simple to code, even for complex problems[7] .

\subsection{Tabu Search}

Tabu search is a meta-heuristic that guides a local exploration process to search the result space further than local optimality. TS avoid entrapment in local minima and continues the search to give a near optimal final solution. TS is very general and conceptually much simpler than other meta heuristic algorithms such as genetic algorithm, simulated annealing and ant colony optimization algorithms. TS does not require special memory space. TS takes short searching time to solve combinatorial optimization problems. TS uses specific set of constraints, known as tabu conditions, in order to avoid blind search.

\subsection{Ant Colony Optimization}

ACO is versatile and can be applied to similar versions of the same problem; for example, there is a straightforward extension from the Traveling Salesman Problem (TSP) to the Asymmetric Traveling Salesman Problem (ATSP). It is robust and can be applied with only minimal changes to other combinatorial optimization problems such as the Quadratic Assignment Problem (QAP) and the Job-Shop Scheduling Problem (JSP).

\subsection{Memetic Algorithm}

Memetic algorithm can handle complex objective functions. It combines the advantages of local search and genetic algorithm for optimization problems.MA can be used for global search. It is based on a genetic algorithm and extended by a search technique to further improve in dividual fitness that may keep with the population diversity and reduce the likelihood premature convergence.

Table 1: Comparison of different heuristic approaches

\begin{tabular}{|c|c|c|c|c|c|c|}
\hline $\begin{array}{l}\text { Heuristic } \\
\text { Approaches }\end{array}$ & Makespan & Cost & Parameters & Services & $\begin{array}{l}\text { Local/Global } \\
\text { Search }\end{array}$ & $\begin{array}{l}\text { Optimization } \\
\text { Problems }\end{array}$ \\
\hline Tabu Search & High & High & $\begin{array}{l}\text { Less } \\
\text { Parameters }\end{array}$ & $\begin{array}{l}\text { Simpler, easy to } \\
\text { implement, no special } \\
\text { memory required }\end{array}$ & Local search & $\begin{array}{l}\text { Combinatorial } \\
\text { Optimization }\end{array}$ \\
\hline Hill Climbing & High & High & Less & $\begin{array}{l}\text { Simpler, straight } \\
\text { forward }\end{array}$ & Local search & Simple optimization \\
\hline $\begin{array}{l}\text { Simulated } \\
\text { Annealing }\end{array}$ & High & Moderate & Less & $\begin{array}{l}\text { Easy to code, robust } \\
\text { heuristic }\end{array}$ & Local search & $\begin{array}{l}\text { Combinatorial } \\
\text { Optimization }\end{array}$ \\
\hline $\begin{array}{l}\text { Genetic } \\
\text { Algorithm }\end{array}$ & Moderate & High & $\begin{array}{l}\text { More } \\
\text { Function }\end{array}$ & Easy to implement & Global Search & $\begin{array}{l}\text { Multi objective } \\
\text { optimization }\end{array}$ \\
\hline $\begin{array}{l}\text { Memtic } \\
\text { Algorithm }\end{array}$ & Moderate & Moderate & $\begin{array}{l}\text { More } \\
\text { function }\end{array}$ & Flexible & Global search & $\begin{array}{l}\text { Complex objective } \\
\text { function, combinatorial }\end{array}$ \\
\hline
\end{tabular}




\begin{tabular}{|l|l|l|l|l|l|l|}
\hline $\begin{array}{l}\text { Ant Colony } \\
\text { Optimization }\end{array}$ & Moderate & High & Less function & Versatile, robust & Global & $\begin{array}{l}\text { Static and Dynamic } \\
\text { optimization }\end{array}$ \\
\hline Hybrid Heuristic & Low & Low & $\begin{array}{l}\text { More } \\
\text { functions }\end{array}$ & Flexible & Global & Stochastic optimization \\
\hline Hyper-Heuristic & Low & Low & $\begin{array}{l}\text { More } \\
\text { function }\end{array}$ & $\begin{array}{l}\text { Flexible, robust } \\
\text { No. of meta heuristic } \\
\text { required }\end{array}$ & Global & $\begin{array}{l}\text { Real world } \\
\text { optimization, complex }\end{array}$ \\
\hline
\end{tabular}

\section{CONCLUSION AND FUTURE SCOPE}

This paper includes the comparison of various Heuristic approaches. This paper consist the most significant concept of Grid Computing correlated to Scheduling problems. The paper identifies the different types of scheduling based on different criteria. The study exposed the difficulty of the scheduling problem in computational Grid when evaluate to scheduling in parallel and distributed system.

In Future, this work can be done by using Bacterial Foraging Optimal algorithm. Further significant issues are associated to data-aware development. Generally existing Grid approach are task-oriented or resource-oriented approach.

\section{REFERENCES}

[1] Abraham Duarte, Rafael Mart'," Tabu search and GRASP for the maximum diversity problem", Received 22 August 2005; accepted 25 January 2006 Available online 20 March 2006.

[2] Anu," Hybridizing Genetic Algorithm with Hill Climbing in Replacement Operator", Volume 3, Issue 12, December 2013 ISSN: 2277 128X.

[3] Foster, I. and Kesselman, C.,"The Grid: Blueprint for a Future Computing Infrastructure", Morgan Kaufmann Publishers, USA, 2004.

[4] Gopesh Joshi," Review of Genetic Algorithm: An Optimization Technique", Volume 4, Issue 4, April 2014 ISSN: 2277 128X

[5] Lee, A. and Parashar, M., Senior member of IEEE," A Survey of Job Scheduling and Resource Management in Grid Computing", 2008.

[6] Buyya, R. and Venugopal, S.,"A Gentle Introduction to Grid Computing and Technologies" Computer Society of India, july 2005.

[7] Bhuyan, P., Sharma, R., Soni, V.K. and Mishra, M.K.," A Survey of Job Scheduling and Resource Management in Grid Computing", World Academy of Science and Engg And Tech,2010.

[8] Burke,E.K., Hyde,M., Kendall, G., Ochoa, G., Ozcan,E. and Qu, R., "Hyper-heuristics: A survey of the State of the Art",Technical report, University of Nottingham, 2009.

[9] Xhafa, F. and Abraham, A., "Computational models and heuristic methods for Grid scheduling problems" Future Generation Computer System 2010.

[10] Abraham,A., Buyya,R. and Nath,B., "Nature's Heuristics for Scheduling Jobs on Computational Grids". The 8th IEEE Conference on Advanced Computing and Communications, Cochin, India, 2000.
[11] Abraham, I., Aron, R. and Chnna, I.," Hyper-Heuristic Based Resource Scheduling in Grid Environment”, IEEE International Conference on Systems, 2013.

[12] Aron, R. and Channa, I.," Bacterial foraging based hyper-heuristic for resource scheduling in Grid computing", Department of Computer Science and Engineering, Thapar University, Patiala, India, September 2012.

[13] Aron, R. and Chnna, I., "Grid scheduling heuristic methods: State of the Art", ISSN 2150-7988 Volume6 (2014).

[14] Liu, H., Abraham, A. and Hassanien, A.E., "Scheduling jobs on computational grids using a fuzzy particle swarm optimization algorithm", Future Generation Computer Systems, 2010.

[15] Pooranian, Z., Harounabadi, A. and Hedayat, N., "New hybrid Algorithm For Task Scheduling in Grid Computing to Decrease missed Task", world academy of Science, Engg and Tech,2011.

[16] Passino, K.M.,"Biomimicry of Bacterial Foraging for Distributed Optimization and Control",IEEE Control and System Magazine, 2002.

[17] Richa Garg, Saurabh mittal," International Journal of Advanced Research in Computer Science and Software Engineering", Volume 4, Issue 4, April 2014 ISSN: 2277 $128 \mathrm{X}$.

[18] Garg, S., Konugurthi, P.and Buyya, R.," A linear programming driven genetic algorithm for meta scheduling on utility Grids", in:16th International Conference on Advanced Computing and Communication, ADCOM 2008, IEEE Press, New York, USA, 2008.

[19] Hu, M. and Verravalli, B., Senior Member of IEEE, "Requirement-Aware scheduling of Bag-of-tasks applications on Grid with Dynamic Resilience”, 2013.

[20] Carretero, J. and Xhafa,F. and abraham,A. "Genetic Algorithm based Schedulers for Grid Computing Systems", International Journal of Innovative Computing, Information and Control, Vol 3, No.6, 2007. 